\title{
AVALIAÇÃO CLÍNICA E DE SINTOMAS PSIQUIÁTRICOS NO HIPOTIREOIDISMO SUBCLÍNICO
}

Patrícia de Fátima dos Santos Teixeira*, Vaneska Spinelli Reuters, Cloyra Paiva Almeida, Márcia Martins ferreira, Márcia Branco Wagman, fabíola Alves Aaráo Reis, Antonio José leal Costa, Mário Vaisman

Serviços de Endocrinologia e de Psicologia Médica do Hospital Universitário Clementino Fraga Filho (HUCFF); Núcleo de Estudos de Saúde Coletiva (NESC) da UFRJ; Faculdade de Medicina-UFRJ, Rio de Janeiro/RJ

\author{
*Correspondência: \\ Av. Canal de Marapendi, \\ 1300/1909 \\ Barra da Tijuca, Rio de \\ Janeiro, RJ \\ Tel: (21) 2484-8950 \\ patricia.hu@terra.com.br
}

\section{RESUMO}

OBjetivo. Analisar e correlacionar características clínicas, sintomas psiquiátricos e dados laboratoriais no hipotireoidismo subclínico $(H S)$.

Métodos. Estudo transversal comparando achados de 103 pacientes com HS aos de 60 indivíduos eutireoidianos. A avaliação clínica e a psiquiátrica foram baseadas, respectivamente, na escala de Zulewski e nos questionários de Hamilton A, Hamilton D e Beck. Todos foram submetidos a dosagens de tireotropina (TSH), T4 livre e antitireoperoxidase (ATPO). Variáveis contínuas foram analisadas por meio do teste $t$ de Student, quando de distribuição normal, e dos testes de Mann-Whitney e Kruskal Wallis, quando "não normais". Variáveis categóricas por meio dos testes Qui-quadrado, exato de Fisher e Kruskal Wallis. Análise multivariada foi utilizada para estudo de variáveis de confundimento.

Resultados. O nível médio de TSH, no HS, foi 7,76 $\pm 2,9 \mu \mathrm{UI} / \mathrm{mL}$ e I,66 $\pm 0,6 \mu \mathrm{UI} / \mathrm{mL}$ nos eutireoideanos ( $p=0,00 \mathrm{I}$ ). $\mathrm{O}$ nível de T4L foi menor no HS, apresentando correlação linear negativa com TSH. Ocorreu maior freqüência de escore clínico anormal (48,3 vs 67\%; $p=0,020)$, de sintomas de depressão, pela escala de Beck no HS $(20,5$ vs 44,2\%; $p=0,0$ I I) e de sintomas de ansiedade ( 86 vs $63,4 \% ; p=0,004$ ) no HS. A presença de sintomas de depressão e ansiedade associouse de forma positiva com pontuação no escore clínico e níveis de TSH. Não houve associação entre achados clínicos ou psiquiátricos e etiologia do HS, presença de ATPO, idade ou menopausa.

Conclusäo. $O$ estudo aponta para associaçãa entre $\mathrm{HS}$, achados clínicos e sintomas psiquiátricos. Ensaios clínicos são necessários para avaliar uma possível melhora com levotiroxina.

Unitermos: Hipotireoidismo. Sintomas. Depressão. Ansiedade.

\section{INTRODUÇÃO}

Hipotireoidismo subclínico (HS) é definido pela elevação do nível sérico de hormônio tireoestimulante (TSH), acima do limite superior da normalidade, associada a níveis normais de tiroxina livre $(\mathrm{T} 4 \mathrm{~L})^{1,2,3}$.

Sua prevalência é de $1 \%$ a $10 \%$ em adultos, em diferentes estudos ${ }^{4-8}$. Há aumento na prevalência com a progressão da idade, chegando a valores próximos a 20\% em mulheres com mais de 60 anos².

Ensaios clínicos randomizados demonstram um aumento na prevalência de sintomas de hipotireoidismo em indivíduos com HS, quando da análise dos resultados basais $1,9,10$. Um estudo de prevalência com 25.862 adultos demonstrou que queixas clínicas são mais freqüentes nos pacientes com HS que nos eutireoidianos, porém menos freqüentes que nos pacientes com hipotireoidismo manifesto $(H M)^{\prime \prime}$. Vale ressaltar que, nesse estudo, foram incluídos pacientes em uso de levotiroxina (LT4) e que não foi utilizada dosagem de T4L como critério diagnóstico de HS (somente TSH e T4 total), mesmo em mulheres em reposição estrogênica". Outros estudos transversais e do tipo casocontrole não confirmam essas observações 1,12,13. Também não existem evidências suficientes para indicar-se o uso de levotiroxina para queixas clínicas, já que diferentes estudos de intervenção demonstram dados conflitantes $1,2,10$.
Monzani, em 1999, relatou freqüência significativamente maior de sintomas neuromusculares (parestesia, cãimbras, fadiga e fraqueza muscular) em pacientes com HS comparados a um grupo controle eutireoideano, e que os sintomas correlacionavam-se a maiores níveis de TSH. Depois da terapia com LT4, houve melhora' ${ }^{14}$.

Entre as manifestações psiquiátricas descritas em pacientes com hipotireoidismo subclínico encontram-se distúrbios do humor, modificações na evolução dos distúrbios depressivos, assim como a resposta desses às medicações antidepressivas, além de vários graus de déficits cognitivos ${ }^{14-19}$. Também não existe consenso se a terapia com levotiroxina traria benefícios a esses pacientes!.

Baseando-se na divergência encontrada na literatura, propusemos o presente estudo, com o objetivo de descrever as características clínicas e laboratoriais basais de pacientes com HS e avaliar a associação entre HS e achados clínicos e/ou sintomas psiquiátricos por meio da comparação com os achados de pacientes eutireoidianos. Também objetivamos avaliar a associação entre níveis de TSH, ou positividade de anticorpo antitireoperoxidase (ATPO), e pontuação no escore clínico e/ou sintomas psiquiátricos. 


\section{Métodos}

\section{Desenho do estudo}

Trata-se de um estudo transversal com análise dos achados clínicos, psiquiátricos e laboratoriais basais encontrados em pacientes com hipotireoidismo subclínico e comparação aos de indivíduos eutireoidianos.

\section{Pacientes e avaliações}

No período de fevereiro/200 I a fevereiro/2004, foram encaminhados $|6|$ pacientes ao ambulatório de Endocrinologia do Hospital Universitário Clementino Fraga Filho (HUCFF) da Universidade Federal do Rio de Janeiro (UFRJ), para avaliação dos seguintes critérios de inclusão no estudo:

- Idade $\geq 18$ e $\leq 70$ anos;

- Duas dosagens de TSH com resultados acima do limite superior da normalidade $(>4,0 \mu \mathrm{UI} / \mathrm{mL})$ e com intervalo de 6 semanas entre elas;

- Dosagem de T4 livre dentro da faixa da normalidade (0,8-1,9ng/dL);

- Uma das dosagens hormonais, necessariamente, realizada no Laboratório de Patologia Clínica do HUCFF;

- No caso de hipertireoidismo prévio, confirmação de estado de eutireoidismo laboratorial, por um ano, antes da evolução para HS;

- Não apresentar doenças ou usar drogas que interfiram na função tireoidiana (incluindo levotiroxina).

O recrutamento ocorreu após divulgação aos serviços médicos e solicitações de encaminhamento dos casos prevalentes. Também foi realizada busca ativa no serviço de análises clínicas do HUCFF, frente aos resultados de TSH e T4L dosados em 200 I. No mesmo período, foram selecionados pacientes eutireoidianos, acompanhados nos diferentes ambulatórios do HUCFF, obedecendo aos seguintes critérios de inclusão:

- Idade $\geq 18$ e $\leq 70$ anos;

- Dosagens de TSH e T4 livre com resultados nos limites da normalidade $(0,4-4,0 \mu \mathrm{UI} / \mathrm{mL}$ e 0,8 - I,9 ng/dL);

- Pesquisa negativa de anticorpo antitireoperoxidase (ATPO);

- Dosagens hormonais e pesquisa de ATPO, necessariamente, realizados no Laboratório de Patologia Clínica do HUCFF;

- Exame clínico da glândula tireóide dentro dos limites da normalidade;

- Não apresentar história de doença tireoidana;

- Não apresentar doenças ou usar drogas que interfiram na função tireoidiana.

Todos os participantes do estudo foram submetidos a anamnese, exame físico e preenchimento de questionário específico, incluindo pesquisa de sinais e sintomas de hipotireoidismo pela escala de Zulewski modificada de Billewicz, com pontuação individual20. Tal preenchimento foi realizado por médicos endocrinologistas participantes da pesquisa, que participam do Ambulatório de Endocrinologia do HUCFF. Para cada sintoma ou sinal presente foi dado um ponto totalizando-se um máximo de 12 pontos $^{20}$.

Pontuações de escore acima de cinco pontos demonstram forte correlação com hipotireoidismo manifesto e entre zero e dois são consideradas normais. Valores na faixa de três a cinco são compatíveis com disfunção tireodiana subclínica ${ }^{20}$. No presente estudo, foram consideradas anormais as pontuações maiores que dois (incluindo-se resultados compatíveis com HS e HM). Receberam ponto apenas os sintomas presentes no período de um ano anterior à pesquisa; queixas crônicas não foram valorizadas por não poderem ser atribuídas à disfunção tireoideana, exceto aquelas que se agravaram significativamente no mesmo período. No trabalho original de Zulewski, foi adicionado um ponto para as mulheres com idade inferior a 55 anos, com o objetivo de equilibrar estatisticamente os grupos, uma vez que a freqüência desses sintomas e sinais foi significativamente maior nas mulheres com 55 anos ou mais ${ }^{20}$.

Todos os participantes do estudo assinaram termo de consentimento livre e esclarecido aprovado pelo Comitê de Ética em Pesquisa do HUCFF/ Faculdade de Medicina da UFRJ ( $n^{\circ} 0$ I2/0 I). Para participarem da avaliação psiquiátrica, foram excluídos os indivíduos que não sabiam ler ou escrever e aqueles em uso de medicações psicotrópicas.

As dosagens laboratoriais, que foram objeto de análise no estudo, foram realizadas a partir de coleta de sangue, no período da manhã, no Laboratório de Patologia Clínica do HUCFF.

Todas as avaliações psiquiátricas foram realizadas pelo mesmo psiquiatra, participante da pesquisa, por meio da aplicação dos questionários: Hamilton A e Hamilton D. Durante a entrevista psiquiátrica, os pacientes também preencheram o questionário auto-aplicável de Beck, para sintomas de depressão. Considerou-se o paciente portador de sintomas de ansiedade quando sua pontuação na escala de Hamilton $A$ foi > 5; e portador de sintomas de depressão quando, na escala de Hamilton D, sua pontuação foi $\geq 8$ ou, na escala de Beck, sua pontuação foi $\geq 10^{21-24}$.

\section{Ensaios laboratoriais}

TSH dosado por quimioluminescência de terceira geração, kit DPC ${ }^{\circledR}$ (Diagnostic Products Corporation) e aparelho Immulite $200{ }^{\circledR}$. Valores de referência: 0,4 - 4,0 $\mu \mathrm{UI} / \mathrm{mL}$ e variações intra-ensaio e interensaio, respectivamente, de 3,8\% - 12,5\% e 4,6\% - $12,5 \%$.

T4L dosado por quimioluminescência, kit DPC ${ }^{\circledR}$ e aparelho Immulite $2000^{\circledR}$. Valores de referência: 0,8 - I,9 ng/dl e variaçōes intraensaio e interensaio, respectivamente, de 4,4\% - 7,5\% e 4,8\% - 9,0\%.

ATPO dosado por quimioluminescência, kitDPC ${ }^{\circledR}$, aparelho Immulite $2000{ }^{\circledR}$. Valores de normalidade $\leq 35 \mathrm{UI} / \mathrm{mL}$ e variações intra-ensaio e interensaio, respectivamente, de 4,3\%-5,6\% e 7,8 \% - 10,5\%.

\section{Análise estatística}

Foi utilizado o programa SPSS, versão I0, para a análise estatística. O nível de significância estatística adotado foi de 5\%. As variáveis contínuas foram analisadas por meio dos testes t de Student e de MannWhitney, para comparações de médias de distribuição "normal" e "não normal", respectivamente ${ }^{25}$. A única variável contínua estudada considerada como de distribuição normal foi a idade. O teste de MannWhitney também foi utilizado para comparação de variáveis ordinais em dois grupos de estudo ${ }^{25}$. Para comparação de proporções entre dois grupos foram utilizados os testes Qui-quadrado e exato de Fisher e o teste de Kruskal Wallis foi utilizado para comparações entre três ou mais grupos ${ }^{25}$. Foram realizadas regressões lineares para avaliação da 


\begin{tabular}{|c|c|c|c|c|}
\hline \multicolumn{2}{|c|}{ Tipo de paciente/achado clínico } & \multirow{2}{*}{$\begin{array}{c}\text { HS } \\
37,9 \% \\
43,7 \% \\
8,7 \%\end{array}$} & \multirow{2}{*}{$\begin{array}{c}\text { Eutireoidiano } \\
25,9 \% \\
22,4 \% \\
6,9 \%\end{array}$} & \multirow{2}{*}{$\begin{array}{r}\text { p valor* } \\
0,121 \\
0,007 \\
0,680\end{array}$} \\
\hline & $\begin{array}{l}\text { Pele seca } \\
\text { Parestesias } \\
\text { Diminuição da sudorese }\end{array}$ & & & \\
\hline Sintomas & $\begin{array}{l}\text { Constipação } \\
\text { Ganho de peso } \\
\text { Rouquidão } \\
\text { Diminuição da audiçãa } \\
\text { Lentidão de movimentos } \\
\text { Pele espessa }\end{array}$ & $\begin{array}{l}15,5 \% \\
31,1 \% \\
34,0 \% \\
15,5 \% \\
1,0 \% \\
27,2 \%\end{array}$ & $\begin{array}{l}10,3 \% \\
48,3 \% \\
17,2 \% \\
12,6 \% \\
0,0 \% \\
13,8 \% \\
\end{array}$ & $\begin{array}{c}0,350 \\
0,030 \\
0,023 \\
0,6 \\
0,45 \\
0,05\end{array}$ \\
\hline Sinais & $\begin{array}{l}\text { Pele fria } \\
\text { Puffnessperiorbitário } \\
\text { Diminuição do reflexo aquileu }\end{array}$ & $\begin{array}{l}6,8 \% \\
41,7 \% \\
12,6 \%\end{array}$ & $\begin{array}{l}5,2 \% \\
24,6 \% \\
8,8 \%\end{array}$ & $\begin{array}{l}0,68 \\
0,03 \\
0,46\end{array}$ \\
\hline
\end{tabular}

*Qui-quadrado, Pearson

influência da idade e níveis de TSH, nos níveis de T4L, bem como da idade nos níveis de TSH. Análise multivariada foi utilizada, no modelo de regressão linear, para avaliação da interferência dessas três variáveis entre $\mathrm{si}^{25}$. Também foi utilizada análise multivariada, no modelo de regressão logística, para avaliação da influência da menopausa na associação entre $\mathrm{HS}$ e pontuações anormais no escore clínico ${ }^{25}$.

A variável TSH, originalmente medida numa escala contínua, foi reduzida a uma escala ordinal, com três níveis (faixa I: $>4,0-8,0$ $\mu \mathrm{UI} / \mathrm{mL}$; faixa 2: >8,0- I2 $\mu \mathrm{UI} / \mathrm{mL}$ e faixa 3: $>12,0 \mu \mathrm{UI} / \mathrm{mL})$. 0 teste de Kruskal Wallis foi utilizado para avaliação da distribuição de variáveis contínuas de distribuições "não normais" em três ou mais grupos de estudo 25 .

\section{Resultados}

Foram incluídos 103 pacientes com HS (97 mulheres) e 60 indivíduos eutireoidianos (58 mulheres). O surgimento do HS foi espontâneo em $84,5 \%$ ( $n=87$ ) dos casos, conseqüência de tratamento de hipertireoidismo em 6,8\% ( $n=7)$ e de tratamento cirúrgico de doença nodular benigna da tireóide em 7,8\% $(n=8)$. A idade média dos pacientes foi 49,5 \pm 10,8 anos e dos indivíduos eutireoidianos foi $44,5 \pm 9,3$ anos $(p=0,003)$.

Entre as pacientes com HS, observou-se maior freqüência $(84,3 \%)$ de menopausa do que entre as eutireoidianas $(68,7 \%)$, com $p=0,020$.

Não foram encontradas diferenças entre as freqüências $(7,4 \%$ nas pacientes com HS e 8,8\% nas eutireoidianas) de mulheres que fazem terapia de reposição estrogênica nos dois grupos $(p=0,075)$.

O nível médio de TSH foi 7,76 $\pm 2,9 \mu \mathrm{Ul} / \mathrm{mL}$ nos pacientes com $\mathrm{HS}$ e I,66 $\pm 0,6 \mu \mathrm{UI} / \mathrm{mL}$ nos eutireoidianos $(p<0,00 \mathrm{I})$.

O nível médio de T4L demonstrou-se mais baixo nos pacientes $(\mathrm{I}, 08 \pm 0,2 \mathrm{ng} / \mathrm{dL})$ em relação aos eutireoidianos ( $1,25 \pm 0,2 \mathrm{ng} / \mathrm{dL})$, com valor de $p<0,001$. No modelo de regressão linear, evidenciouse correlação negativa entre níveis de TSH e T4L $(p<0,00$ I), sendo o coeficiente de regressão (beta) igual a -0,436. Detectou-se, portanto, que ocorre uma diminuição de cerca de $0,436 \mathrm{ng} / \mathrm{dL}$ no nível de T4L para cada elevação de I,0 $\mu \mathrm{UI} / \mathrm{mL}$ no nível de TSH.

Observou-se, por regressão linear, que também ocorre queda nos níveis de T4L com aumento da idade, sendo o coeficiente de regressão (beta) igual a $-0,154(p=0,051)$.

Em análise multivariada, observou-se que os níveis de TSH e T4L correlacionam-se negativamente, de forma independente, sem influência da variável idade, com coeficiente beta de $-0,42(p<0,001)$. Não foi observada associaçãa, independente do TSH, entre idade e nível de T4 livre, com coeficiente beta de $-0,082(p=0,258)$. Observou-se que ocorre elevação de $0,172 \mu \mathrm{Ul} / \mathrm{mL}$ (coeficiente de regressão beta), nos níveis de TSH para cada aumento anual da idade $(p=0,028)$.

Evidenciou-se maior freqüência de escore clínico anormal $(\geq 3)$ no HS (67\%) em relação aos eutireoidianos (48,3\%), com valor de $p=0,020$. A pontuação média do escore também foi maior no HS $(3,4 \pm 1,7$ vs $2,8 \pm 1,7)$ com valor de $p=0,030$. $\mathrm{Na}$ análise da distribuição nas diferentes faixas de escore clínico (normal, compatível com HS e compatível com HM), encontramos as seguintes freqüências, respectivamente, no HS: 33\%, 56,3\% e 10,3\%. Nos eutireoidianos, essas freqüências foram: $51,7 \%, 37,9 \%$ e 10,3\%. Na comparação (Mann-Whitney) das diferentes freqüências nas faixas de escore entre os dois grupos, observamos uma significância estatística de 4,8\%.

A distribuição das freqüências de sinais e sintomas da escala de Zulewski entre os dois grupos de pacientes encontra-se na Tabela I. Observa-se que o sintoma mais freqüente foi parestesia, e o sinal mais freqüente foi a presença de puffness periorbitário (definido pela perda da curvatura do osso malar).

Não ocorreu associação entre escore anormal e presença de menopausa e, em análise multivariada, o escore alterado continua associando-se à presença de hipotireoidismo subclínico, independente da presença ou ausência da menopausa. Entre as mulheres com escore anormal, 57,6\% encontram-se na menopausa contra $47 \%$ entre as com escore $<3(p=0,2 \mid 0)$.

A idade média entre mulheres com escore anormal foi $48,5 \pm$ 9,7 anos, sem diferenças, do ponto de vista clínico ou estatístico $(p=0,251)$, à idade média entre as mulheres com escore normal (46,5 \pm II,6 anos).

O nível de TSH entre os pacientes com HS e escore anormal foi 7,7 $\pm 2,7 \mu \mathrm{UI} / \mathrm{mL}$ contra 7,8 $\pm 3,2 \mu \mathrm{UI} / \mathrm{mL}$ nos pacientes com HS e escore normal $(p=0,986)$. 


\begin{tabular}{|c|c|c|c|c|}
\hline & $\begin{array}{c}\text { Ansiedade } \\
\text { (Hamilton A) }\end{array}$ & $\begin{array}{c}\text { Depressão } \\
\text { (Hamilton D) }\end{array}$ & $\begin{array}{l}\text { Depressão } \\
\text { (Beck) }\end{array}$ & $\begin{array}{l}\text { Escore } \\
\text { anormal }\end{array}$ \\
\hline $\begin{array}{c}\text { Faixa } 0 \\
\text { TSH normal } \\
(0,4-4,0 \mu \mathrm{Ul} / \mathrm{mL})\end{array}$ & $63,4 \%$ & $19,6 \%$ & $20,5 \%$ & $51,7 \%$ \\
\hline $\begin{array}{c}\text { Faixa I } \\
(>4,0-8,0 \mu \mathrm{Ul} / \mathrm{mL})\end{array}$ & $83,0 \%$ & $21,4 \%$ & $38,9 \%$ & $31,3 \%$ \\
\hline $\begin{array}{c}\text { Faixa } 2 \\
(>8,0-12,0 \mu \mathrm{Ul} / \mathrm{mL})\end{array}$ & $92,0 \%$ & $36,0 \%$ & $52,0 \%$ & $34,6 \%$ \\
\hline $\begin{array}{c}\text { Faixa } 3 \\
(>12,0 \mu \mathrm{Ul} / \mathrm{mL}) \\
\text { p. valor* }\end{array}$ & $\begin{array}{l}87,5 \% \\
0,026\end{array}$ & $\begin{array}{l}25,0 \% \\
0,463\end{array}$ & $\begin{array}{l}57,1 \% \\
0,042\end{array}$ & $\begin{array}{l}40,0 \% \\
0,127\end{array}$ \\
\hline
\end{tabular}

* Kruskal Wallis

No estudo psiquiátrico, foram avaliados 89 pacientes com HS e 36 eutireoidianos. Observou-se maior prevalência de sintomas de ansiedade e de depressão (pela escala de Beck) no HS. De acordo com a escala de Hamilton A, 86\% dos pacientes com HS e $63,4 \%$ dos eutireoidianos encontravam-se com pontuações acima do ponto de corte $(p=0,043)$. A intensidade dos sintomas ansiosos foi maior no HS, com pontuação média de $12,7 \pm 6,3$ contra $9,1 \pm 7,2$ nos eutireoidianos $(p=0,003)$. Na escala de Beck, observamos a presença de sintomas de depressão em $44,2 \%$ dos pacientes contra $20,5 \%$ nos eutireoidianos $(p=0,0 \mathrm{I} \mid \mathrm{l})$. A pontuação média obtida nessa escala foi de 9,5 $\pm 7,1$ no HS e 6,8 \pm 7,3 nos eutireoidianos ( $p=0,0$ । 3). Na escala de Hamilton D, a freqüência de pontuações anormais no HS também foi maior $(25,8 \%)$ que no grupo eutireoidiano $(19,4 \%)$, porém sem significância estatística $(p=0,448)$. A pontuação obtida, por essa escala, foi maior no HS (5,3 $\pm 3,7$ vs $3,8 \pm 7,3 \pm 3,5)$ com $p=0,032$; porém situando-se abaixo do ponto de corte utilizado.

Não foram observadas diferenças entre pacientes com HS espontâneo ou secundário nas freqüências de escore clínico anormal (42,7\% vs $54,5 \%, p=0,459)$, de sintomas de ansiedade $(86,3 \%$ vs $83,3 \%$, $p=0,678)$, sintomas de depressão por Hamilton D $(24,7 \%$ vs $33,3 \%$, $p=0,524)$ ou sintomas de depressão pela escala de Beck $(31,8 \%$ vs $40 \%, p=0,533)$.

No estudo por faixas de TSH, não ocorreu associação entre as diferentes faixas e freqüências de escore clínico anormal, conforme Tabela 2.

Detectou-se maiores freqüências de sintomas de depressão, com a elevação dos níveis de TSH e maiores freqüências de sintomas de ansiedade com elevação do TSH até o nível de $12 \mu \mathrm{UI} / \mathrm{mL}$. Porém não foram encontradas diferenças com significância estatística nas diversas pontuações médias obtidas nas escalas de sintomas depressivos à medida que ocorre elevação do TSH, por faixas, no HS. Na escala de sintomas de ansiedade, observou-se pontuação média de 12,9; 12,3 e 12,5 nos pacientes com HS distribuídos nas faixas I, 2 e 3 , respectivamente $(p=0,887)$. Na escala de Beck, tal pontuação foi 9, I; 9 e I4,I ( $p=0,507)$ e, na Hamilton D, 5,3; 5,5 e $4,3(p=0,878)$, ambas obedecendo a mesma sequêencia de distribuição por faixas de TSH.
Ao se avaliar os níveis médios de TSH nas diferentes faixas de escore também não ocorreram diferenças com significado estatístico ou clínico. Os níveis médios de TSH foram, respectivamente: 4,9,6 e $5,6 \mu \mathrm{Ul} / \mathrm{mL}(p=0,158)$. Em relação aos níveis de T4L, nas diferentes faixas de escore clínico, observou-se que, à medida que aumentou a pontuação no escore, ocorreu queda nos níveis médios de T4L, porém sem significância estatística. Os valores médios encontrados de T4L foram, respectivamente: I, I8; I, I 4 e I,05 ng/dL $(p=0,073)$. O nível de significância estatístico foi próximo a 5\% $(p=0,088)$ quando na comparação dos níveis médios de T4L nos subgrupos de escore normal e escore compatível com HM (>5).

No subgrupo de pacientes com HS, 60,2\% apresentam pesquisa positiva de ATPO, porém não foi evidenciada associação entre a presença do anticorpo e escore clínico anormal. Escore clínico anormal esteve presente em 64,5\% dos pacientes com HS e ATPO circulante, e em $73,7 \%$ dos sem ATPO ( $p=0,340)$. A freqüência de escore clínico compatível com HM (>5) foi II ,3\%, quando a pesquisa de ATPO foi positiva e $10,5 \%$ quando negativa $(p=0,601)$.

A presença de ATPO, nos pacientes com HS, também não aumentou a freqüência de sintomas de ansiedade ou depressão. Sintomas de ansiedade estiveram presentes em $84,9 \%$ dos pacientes com HS e ATPO circulante e em 87, I\% dos sem o anticorpo $(p=0,782)$. Sintomas de depressão, por Beck, foram freqüentes em $41,5 \%$ dos com anticorpo e em $48,4 \%$ dos demais $(p=0,540)$. Observou-se que os pacientes com HS e pesquisa negativa de ATPO apresentam maior freqüência de sintomas de depressão, pela escala de Hamilton D (4I,9\% vs |6, | \%, $p=0,008)$.

O nível médio de TSH foi maior ( $8,05 \mathrm{vs} 7,22 \mu \mathrm{UI} / \mathrm{mL}$ ) e de T4L foi menor ( 1,06 vs $1,12 \mathrm{ng} / \mathrm{dL}$ ) nos pacientes com HS e anticorpo circulante, porém sem significância estatística $(p=0,170$ e $p=0,160)$.

A presença de sintomas de depressão (pela escala de Beck) e de ansiedade foi mais freqüente nos pacientes com escore anormal, e ocorreu maior freqüência desses sintomas à medida que o paciente apresentou escore clínico mais elevado, conforme Tabela 3. A intensidade dos sintomas de ansiedade foi maior nos pacientes com escore anormal, com pontuações médias de 12,7 $\pm 6,6$ vs $9,9 \pm 6,8(p=0,016)$. A pontuação média obtida nas 


\begin{tabular}{|c|c|c|c|}
\hline Escala/escore clínico & $\begin{array}{c}\text { Ansiedade } \\
\text { (Hamilton A) }\end{array}$ & $\begin{array}{c}\text { Depressão } \\
\text { (Hamilton D) }\end{array}$ & $\begin{array}{c}\text { Depressão } \\
\text { (Beck) }\end{array}$ \\
\hline Anormal $(\geq 3)$ & $85,9 \%$ & $26,7 \%$ & $46,1 \%$ \\
\hline Compatível com HS ( $\geq 3-5)$ & $82,5 \%$ & $24,2 \%$ & $41,9 \%$ \\
\hline $\begin{array}{l}\text { (Normal vs anormal) } \\
\text { p. valor** }\end{array}$ & 0,021 & 0,426 & 0,009 \\
\hline (entre as três diferentes pontuações de escore) & 0,023 & 0,404 & 0,010 \\
\hline
\end{tabular}

*Qui-quadrado, Pearson ** Kruskal Wallis

escalas de sintomas de depressão também foi maior na presença de escore clínico anormal.

$\mathrm{Na}$ escala de Beck, encontrou-se uma média acima do ponto de corte para a presença de sintomas depressivos quando o escore clínico estava anormal $(10,1 \pm 0,9)$, a qual foi significativamente maior $(p=0,004)$ que a encontrada quando o escore clínico era normal $(6,4 \pm 0,9)$. Na escala de Hamilton $D$, a pontuação média também foi maior na presença de escore clínico anormal $(5,5 \pm 0,5$ vs 3,8 \pm 0,5; $p=0,008)$.

Não foram encontradas diferenças, do ponto de vista clínico ou estatístico, entre os indivíduos com ou sem sintomas de depressão, pela escala de Beck, quanto à variável idade ou presença de menopausa. A idade média entre os com sintomas de depressão foi 47,3 \pm 11,5 contra 47,7 \pm 9,5 anos nos sem sintomas, pela escala de Beck $(p=0,844)$. Entre as mulheres com sintomas de depressão, observamos que $51,2 \%$ encontravam-se na menopausa contra 48,6\% entre as sem sintomas de depressão, pela mesma escala $(p=0,792)$.

Também não foram encontradas diferenças $(p=0,608)$ entre a idade média dos participantes com sintomas (47,8 \pm 10,3 anos) ou sem sintomas de ansiedade (46,7 $\pm 9,3$ anos). Não foi detectada diferença $(p=130)$ entre presença $(54,8 \%)$ ou ausência $(45,2 \%)$ de menopausa entre as mulheres com sintomas de ansiedade.

\section{Discussão}

Os resultados divergentes dos estudos que avaliam a associação entre HS e achados clínicos ou laboratoriais decorrem de diferentes metodologias e critérios de inclusão. No presente estudo, só foram incluídos pacientes com HS confirmado após intervalo mínimo de seis semanas, já que alterações transitórias no eixo hipotálamo-hipófisetireóide podem manter níveis anormais de TSH por tal período ${ }^{26}$. Também foram excluídos pacientes que apresentassem situações que interferissem na função tireoidiana ou dosagens laboratoriais e, por isso, apenas 103 pacientes foram incluídos após avaliação inicial de 161 pacientes encaminhados ao Ambulatório de Endocrinologia.

O nível médio de TSH no HS foi de 7,76 $\mu \mathrm{UI} / \mathrm{mL}$, compatível com diferentes estudos de prevalência de disfunção tireoidiana ${ }^{4-8}$.
Dado de relevância visto que estudos que sugerem associações entre achados clínico-laboratoriais e HS encontram maiores evidências a partir da elevação dos níveis de TSH acima de $10 \mu \mathrm{UI} / \mathrm{mL}$, o que não é freqüente no $\mathrm{HS}^{8,26}$.

Os níveis mais baixos de T4L encontrados nos pacientes com HS poderiam sugerir um estado de insuficiência tireoidiana inicial e justificar os achados clínicos e psiquiátricos encontrados. Observou-se uma associação entre HS e queixas clínicas, bem como sintomas de depressão, quando o instrumento utilizado foi o questionário auto-aplicável de Beck. Os pacientes que apresentaram mais queixas clínicas também apresentaram maior freqüência de sintomas de depressão. Como tal dado foi evidenciado pelo questionário auto-aplicável, poderíamos supor que pacientes com HS apresentam mais queixas clínicas e sentem-se mais doentes que os indivíduos sem HS. Também foi evidenciado que pacientes com HS apresentam maior freqüência de sintomas de ansiedade e que esses sintomas associam-se com aumento no escore clínico. A pontuação média na avaliação pela escala de Hamilton A foi significativamente maior no HS, estando acima de duas vezes o valor utilizado para ponto de corte, o que demonstra maior intensidade dos sintomas. Os resultados reforçam a necessidade de realização de ensaios controlados para avaliação dos efeitos do uso de levotiroxina nos achados clínicos e psiquiátricos. Podem ocorrer benefícios com a terapêutica ou ainda exacerbação dos sintomas de ansiedade com uso de LT4 conforme já relatado ${ }^{29}$. Os sintomas psiquiátricos encontrados podem fazer parte de quadros de depressão ou ansiedade, uma vez que podem estar presentes nos dois diagnósticos, e o seu diagnóstico se faz necessário nesses doentes.

Conforme Hollowell, observou-se, por regressão linear, que o T4L diminuiu com a progressão da idade, porém, em análise multivariada, essa associação correlacionou-se de forma dependente com o TSH ${ }^{4}$.

Verificou-se a presença de correlação negativa entre níveis de TSH e T4L, independente da idade. Possivelmente, elevação do TSH trata-se de uma resposta à diminuição nos níveis séricos de T4L, podendo ser um sinal de disfunção tireodiana precoce. Apesar do HS ser mais freqüente em mulheres idosas e ocorrer elevação do TSH com a idade, isso não ocorreu de forma linear direta e independente dos níveis de T4L. 
A presença de sinais e sintomas de hipotireoidismo foi mais comum em pacientes com HS, refletindo-se em maior freqüência de escore clínico anormal entre eles. Tal associação foi independente do fator idade e da presença de menopausa. Como os sinais e sintomas de hipotireoidismo são inespecíficos e a faixa etária mais acometida refere-se ao grupo de mulheres na menopausa, existe uma preocupação quanto à possibilidade de se atribuir sinais e sintomas do climatério à possível disfunção tireoidiana e, com isso, iniciar-se um tratamento desnecessário. Além do custo, é descrito que cerca de $20 \%$ dos pacientes que utilizam T4L encontram-se com níveis de TSH abaixo da normalidade, fator associado com osteoporose e risco de fibrilação atrial',2,11.

Não ocorreu, em análise multivariada, associação entre menopausa e maior frequêencia de escore clínico anormal e este continuou se associando ao HS independente do fato de haver maior freqüência de mulheres na menopausa entre as pacientes com HS. A freqüência de menopausa também foi semelhante entre os grupos com ou sem sintomas psiquiátricos.

$\mathrm{Na}$ análise dos sinais e sintomas, observamos que não só sintomas subjetivos como também diversos sinais foram mais freqüentes nos pacientes com HS em relação aos eutireoidianos. A seleção de pacientes com HS em ambiente hospitalar poderia justificar a maior prevalência de sinais e sintomas, porém a comparação a um grupo eutireoidiano de mesma origem minimiza tal fator de confundimento. Na avaliação clínica, nem os pacientes nem os avaliadores encontravam-se mascarados quanto ao status da função tireoidiana, o que poderia gerar viés de informação. Porém, para minimizar tal fato, somente os mesmos médicos da pesquisa fizeram as avaliações, com padronização entre eles do que seria considerado positivo. Podemos exemplificar o sinal mais freqüente, que foi a presença de puffness infra-orbitário, o qual só foi considerado presente quando ocorreu perda da curvatura do osso malar.

A presença de puffness infra-orbitário foi apontada por Zulewski como um dos sinais com maior valor preditivo positivo para estimativa de hipotireoidismo em um paciente com suspeita clínica, com a vantagem de ser facilmente detectável ${ }^{20}$.

O achado de frequêencias semelhantes de alentecimento do reflexo aquileu nos grupos estudados é bastante discordante dos achados de Zulewski, que considerava este um sinal muito prevalente, específico e de alto valor preditivo para o diagnóstico de hipotireoidismo ${ }^{20}$. Esta diferença talvez possa ser atribuída a fatores tais como a dificuldade em se pesquisar o reflexo em pacientes ansiosos e pouco cooperativos e contar com a colaboração da análise puramente visual.

Não ocorreu associação entre níveis de TSH e escore clínico anormal. Isso poderia ser justificado pelo fato de que se tem descrito que queixas clínicas e musculares se associam com o tempo de instalação de hipotireoidismo, sendo mais freqüente no hipotireoidismo de instalação mais recente e mais rápida do que naqueles com hipotireoidismo de longa data, mesmo com níveis mais elevados de $\mathrm{TSH}^{27}$. Porém, observamos que os pacientes apresentam mais queixas de depressão e de ansiedade à medida que ocorre elevação nos níveis séricos de TSH.
Um possível benefício do tratamento do HS baseia-se na prevenção do surgimento desses sinais e sintomas clínicos quando há evolução para HM, o que ocorre em cerca de $5 \%$, anualmente ${ }^{7,28}$.

Tenta-se preconizar possíveis preditores de progressão de doença, tais como níveis de TSH > $10 \mu \mathrm{UI} / \mathrm{mL}$, presença de ATPO e história prévia de doença tireoidiana!.

No presente estudo, cerca de 60,2\% dos pacientes com HS têm pesquisa positiva de ATPO e poderão apresentar evolução clínica diferente. Os dados aqui presentes correspondem somente aos dados transversais de uma linha de pesquisa que tem continuidade com uma fase de estudo longitudinal concorrente, em que se poderá discutir tal fato. Observamos que esses pacientes com ATPO não apresentam maior freqüência de escore anormal ou sintomas de depressão e ansiedade, além de não apresentarem diferenças do ponto de vista estatístico nos níveis médios de TSH e T4L. Também não ocorreram diferenças nos achados clínicos e psiquiátricos entre pacientes que apresentam HS de origem espontânea ou de origem secundária.

\section{ConClusão}

Os resultados do estudo sustentam a hipótese de associação entre HS, queixas clínicas e sintomas de depressão e ansiedade, independentemente da presença ou ausência de ATPO e da causa do HS. Também não foi evidenciada correlação entre queixas clínicas e níveis de TSH, que estão presentes mesmo quando o nível médio de TSH encontra-se abaixo de $10 \mu \mathrm{UI} / \mathrm{mL}$. Porém, os sintomas de depressão e ansiedade se acentuaram com a elevação nos níveis séricos de TSH. Ensaios clínicos randomizados e controlados com placebo são necessários para avaliar se o tratamento com levotiroxina pode reverter os achados encontrados.

\section{Agradecimentos}

Agradecemos o apoio financeiro do laboratório farmacêutico Sanofi-Aventis para a realização da pesquisa nas fases transversal e prospectiva. Também agradecemos aos alunos do Projeto de iniciação científica que contribuíram na coleta e armazenamento de dados.

Conflito de interesse: Este trabalho contou com apoio financeiro do laboratório Sanofi-Aventis.

\section{SUMMARY}

\section{EVALUATION OF CLINICAL AND PSYCHIATRIC SYMPTOMS IN SUB CLINICAL HYPOTHYROIDISM}

BACKGROUND. This investigation evaluated and correlated clinical, laboratorial aspects and psychiatric symptoms in sub clinical hypothyroidism(SH).

METHODS. Cross sectional study involving 103 patients with SH and 60 subjects without thyroid disease. Clinical and psychiatric evaluation was respectively based on the Zulewski score and Hamilton A, Hamilton D and Beck questionnaires. Serum thyreotropin (TSH), (thyroxine) FT4 and (antithyroperoxidase)ATPO were measured in all participants. Analysis of continuums data was assessed by the Student t- test, for normally distributed data, and by the Mann- Whitney and Kruskal Wallis tests for non-parametric data. The $\chi^{2}$, Fisher's and Kruskal Wallis tests were used to study qualitative variables. Multivariate analyses were used to study confounding variables. 
RESULTS. Meanserum TSHlevelwas $7.76 \pm 2.9 \mu \mathrm{UI} / \mathrm{mL}$ in SH and I.66 $\pm 0.6 \mu \mathrm{UI} / \mathrm{mL}$ in the group without thyroid disease $(p=0.00 \mathrm{I})$. Mean serum T4L was slightly lower among patients with $H S$, and showed a negative linear correlation with TSH. Higher frequencies of abnormal clinical score (48.3 vs. $67.0 \% ; p=0.02)$, depression self reported symptoms (20.5 vs. $44.2 \% ; p=0.01$ I) and anxiety symptoms (86.0 vs. 63.4\%; $p=0.004$ ) occurred more frequently in SH. Frequency of psychiatric symptoms had a positive correlation with the clinical score and serum TSH. There was no association between the clinical and psychiatric findings and the etiology ofSH, presence of ATPO, age or menopause.

CONCLUSIONs. The study showed that $\mathrm{SH}$ was associated with clinical findings and with psychiatric symptoms. Clinical trials are required to evaluate possible improvement with levotiroxine. [Rev Assoc Med Bras 2006; 52(4): 222-8]

KEY wORDs: Hypothyroidism. Symptoms. Depression. Anxiety.

\section{REFERÊNCIAS}

I. Surks MI, Ortiz E, Daniels GH, Sawin CT, Col NF, Cobin RH, et al. Subclinical Thyroid Disease. Scientific Review and Guidelines for Diagnosis and Management. JAMA 2004;29I(2):228-38.

2. Helfand M, U.S. Preventive Services Task Force. Screening for Thyroid Dysfunction in Nonpregnant Adults : A Summary of the Evidence for U.S. Preventive Services Task Force. Ann Intern Med 2004; I 40(2): I 28-4I.

3. Cooper DS. Subclinical hypothyroidism. N Engl J Med 200I; 345(4):260-5.

4. Hollowel JG, Staehling NW, Flanders D, Hannon H, Gunter EW, Spencer CA, et al. Serum TSH,T4, and Thyroid Antibodies in the United States Population (1988 to 1994): National Health and Nutrition Examination Survey (NHANES III). J Clin Endocrinol Metab 2002 ;87(2):489-99.

5. Vanderpump MPJ, Tumbridge VM, French JM, Appleton D, Bates D, Clar $\mathrm{F}$, et al. The incidence of thyroid disorders in the community: a twentyyear follow-up of the Whickham Survey. Clin Endocrinol 1995;43:55-68.

6. Tumbridge WMG, Evered DC, All R, Appleton D, BewisbM, Clark F, Evans JG, et al. The spectrum of thyroid disease in a community: the Whickham survey. Clin Endocrinol (oxf) 1977;7:48I-93.

7. Vanderpump MPJ, Tunbridge W.M. Epidemiology and Prevention of Clinical and Subclinical Hypothyroidism. Thyroid 2002; I 2( I 0): 839-47.

8. Mc Dermott T, Ridgway C. Clinical Perspective. Subclinical Hypothyroidism Is Mild Thyroid Failure and should be treated. J Clin Endocrinol Metab 200 I; 86(I0):4585-90.

9. Meier C, Staub JJ, Roth CB, Guglielmetti R, Kunz M; Miserez A, et al. TSHcontrolled L-thyroxine therapy reduces cholesterol leves and clinical symptoms in subclinical hypothyroidism: A double-bind placebocontrolled trial (Basel Thyroid Study). J Clin Endocrinol Metab 200 I;86(I0):4860-6.

10. Cooper DS, Halpern R, Wood LC, Lewin AA, Ridgway EC. L-thyroxine therapy in subclinical hypothyroidism. Ann Intern Med I 984; I 0 I: 1 8-24.

I I. Canaris GJ, Manowitz NR , Mayor G, Ridgway EC. The Colorado thyroid disease prevalence study. Arch Intern Med 2000; 160:526-34.

12. Lindeman RD, Sachade DS, La Rue A, Romero LJ , Linag HC, Baumgartner RN et al. Subclinical hypothyroidysm in a bioethnic urban community. J Am Geriatr Soc 1999;47:703-9.
13. Bemben DA, Hamm Rm, Morgan L, Winn P, Davis A, Barton E, Thyroid disease in rthe elderly. Part 2: Predictability of subclinical hypothyroidism. J. Fam Pract. 1994:38:583-8.

14. Monzani F, Del Guerra P, Caraccio N, Prunet Ca, Pucci E, Luisi M et al. Subclinical hypothyroidism: neurobehavioral features and beneficial effect of L-thyroxine treatment. Clin Invest 1993;71:367-71 .

15. Baldini M, Vita A, Mauri MC, Amodei V,Carrisi M, Bravin S, et al Psychopathological and cognitive features in subclinical hypothyroidism. Prog Neuropsychopharmacol Biol Psychiatry 1997;2I (6): 925-35.

16. Engum A, Bjoro T, Mykleton A; Dahl AA. An association between depression, anxiety and thyroid function - a clinical fact or an artefact? Acta Psychiatr Scand 2002; 106: 27-34.

17. Gloger S, Fardella C, Santis R, Bitrán J. Relevancia del estudio tireoideo en pacientes con transtornos psiquiátricos. Rev Med Chile 1997; 125: |35|-6.

18. Haggerty JJ Jr, Stern RA, Mason GA, BeckwithJ, Morey CE, Prange AJ Jr. Subclinical Hypothyroidism: A Modifiable Risk Factor for Depression? Am J Psychiatry 1993; I 50(3):508-9.

19. Jackson I. The Thyroid Axis and Depression. Thyroid I 998;8(I0): 95 I-6.

20. Zulewski H, Muller B, Exer P, Miserez AR, Staub JJ. Estimation of tissue hypothyroidism by a new clinical score:evaluation of patients with various grades of hypothyroidism and controls. J clin Endocrinol Metab |997;82:77|-6.

21. Hamilton M: A rating Scale for Depression. J. Neurol Neurosurg Psychiatry 1960;23:56-62.

22. Hamilton M: The assessment of anxiety states by a ratio. Br J. Med Psychol 1959;32:50-55.

23. Lambbert M J, Hatch DR, Kingston Md, Edwards B C. Zung, Beck and Hamilton Scales as measures of treatment outcome: A meta-analytic Comparision. Journal of Consulting and Clinical Psychology 1986; 54(I): 54-59.

24. Zimmerman M, Posternak MA, Chelminsk I Using a self-report depression scale to identify remission in depressed outpatients American Journal of Psichiatry 2004; 16 | (10):1911-13.

25. Luiz RR. Associação Estatística em epidemiologia. In: Epidemiologia. Medronho RA; Carvalho DM; Bloch KV; Luiz RR; Werneck GL. Editora Atheneu. 2003. p.309-334.

26. CHUJ.W, CAPRO L.M. Clinical perspective. The treatment of subclinical hypothyroidism is seldom necessary. J Clin Endocrinol Metab 200 I;86 (I0):459|-9.

27. Monzani F, Caraccio, Siliciano G, Manca L, Murri L, Ferranini E. Clinical and Biochemical Features of muscle dysfunction in subclinical hypothyroidism. J Clin Endocrinol Metab 1997; 82( I0): 33। 5-8.

28. Rosenthal MJ, Hunt W C, Garry PJ, Goodwin JS. Thyroid Failure in the elderly. Microssomal Antibodies as discriminant for therapy. JAMA 1987;258(2): 209-13

29. KONGW.M.; et al. A6-Month Randomized Trial of Thyroxine Treatment in Women with Mild Subclinical Hypothyroidism. Am J Med. 2002, 112:348-354. 